\title{
DOMAINS IN CoPd MULTILAYERS AND THEORETICAL MODELS (Invited)
}

\author{
V. KAMBERSKY \\ Institute of Physics, Acad. Sci. CR, Cukrovarnická 10, 16200 Praha 6, Czech Republic
}

\begin{abstract}
Recent observations of irregular stripe domains in demagnetized thin CoPd multilayers and the comparison of measured domain sizes with" theoretical models are reviewed. The models are discussed in terms of deviations from models of 'equivalent uniform' films, which appear to be small for wide domains (relative to film thickness). Theoretical estimates of significant domain wall energy reduction due to magnetostatic effects, based on micromagnetic simulation, are reported but so far not confirmed experimentally.

KEYWORDS: MULTILAYERS, DOMAINS, WALLS
\end{abstract}

\section{INTRODUCTION}

Stability of stored information as well as (read)write performance are affected by magnetostatic fields depending on the distribution of magnetization. In multilayers, the latter is affected by the spacers; therefore, studies of magnetostatic energy and effective fields have attracted great attention [1-3]. In the present review we wish to stress that the effective fields (unlike the energy densities) in multilayers (ML) are quite close to those in uniform 'equivalent' (UE) films in practically important ranges of domain sizes; hence many previous results concerning recording in uniform films remain approximately valid.

From this viewpoint, the results for isolated cylindrical domains [1] are reviewed, followed by analogous results for stripe domains which allow more intuitive insight. The theory of equilibrium sizes of stripes in arrays [1-4] is reviewed next. Since it is based on the knowledge of wall energy density, the latter is computed, taking into account dipolar interactions, on the basis of micromagnetic simulations [5]. Finally we review a series of experimental results on domains in CoPd ML $[4,6]$.

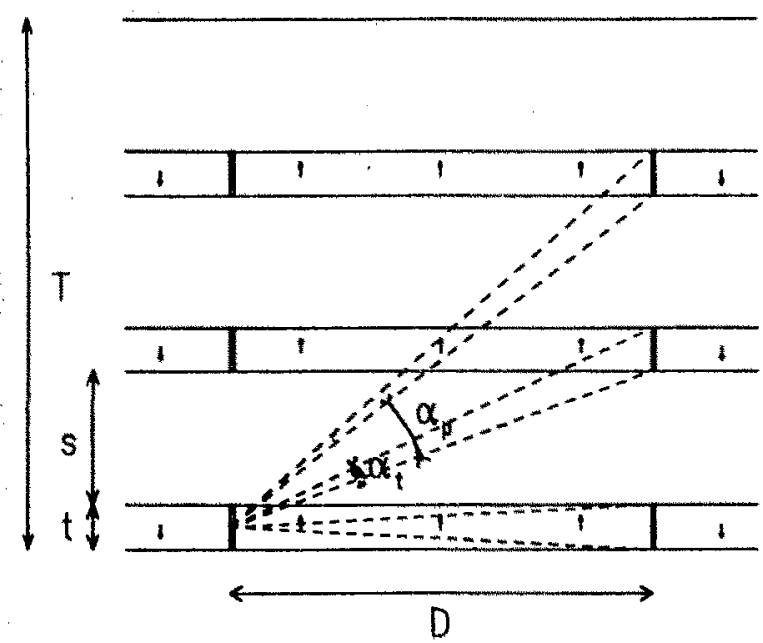

Fig. 1 Stripe-domain model in a ML (and scheme of magnetostatic field calculation in text).

\section{SINGLE DOMAIN MODELS}

Honda, Ikegawa and Kusuda [3] have first pointed out that the computed mean fields, $\mathrm{H}_{\mathrm{dw}}$, acting on walls of isolated cylindrical domains in $\mathrm{ML}$ and tending to expand them, are close to those in UE films when the domains are flat, i.e., their diameter $D$ exceeds the total film thickness, T. In order to define UE films with the same average magnetization $M_{8}^{*}$ and thickness $T$, in analogy to layered crystals, we define $T=N(t+s)$ if $t$ and $s$ denote the thickness of magnetic and spacer layers (Fig. 1) and $\mathrm{N}$ is the number of bilayers.

Fig. 2 illustrates the results [3] as plots of $\mathrm{H}_{d w} / \mathrm{M}_{s}^{*}$ vs. $D / T$ with $M_{s}^{*}=f M_{s}, f=t /(t+s)$ is the magnetic filling factor and $M_{n}$ is the saturation magnetization of magnetic sublayers. The solid and long-dash lines show $\mathrm{H}_{\mathrm{dw}} / \mathrm{M}_{\mathrm{s}}^{*}$ in ML (for $\mathrm{N}=4,10$ and 25) and in UE, respectively. Further, for $\mathrm{N}=10$, the light-dashed lines

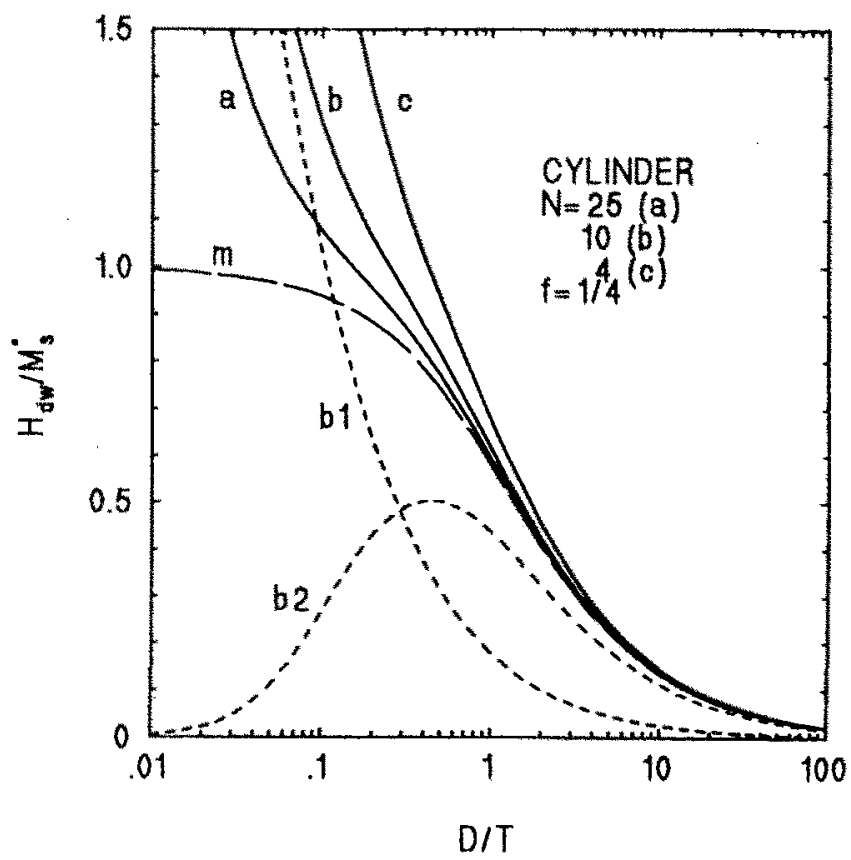

Fig.2 Mean field $H_{\text {tw }}$ at wall of a cylindrical domain of diameter $\mathrm{D}$ in ML of thickness $\mathrm{T}$ (solid). $\mathrm{M}_{*}^{*}$ is the mean magnetization. (b1) and (b2) are the intra- and inter-layer constituents of the (b) line, (m) is $\mathrm{H}_{d w}$ in uniform equivalent film. 
show that also the mixing of two contributions to $\mathrm{H}_{d w}$, from the same magnetic layer $(1$, intra-layer) and from other layers (2, inter-layer), is different in the cases of relatively wide and narrow domains.

For practical purposes, it is useful to note that for $\mathrm{D}>\mathrm{T}$, the UE mean field is well approximated as

$$
\mathrm{H}_{\mathrm{dw}} / \mathrm{M}_{\mathrm{s}}^{*}=(\mathrm{T} / \mathrm{D})[0.59+0.32 \ln (\mathrm{D} / \mathrm{T})]
$$

Fig. 3 shows similar results for isolated stripe domains, which allow to visualize the origin of the main features of these plots: summing up the fields of all magnetic poles in terms of aspect angles at which they are 'seen' from a given point (Fig. 1), we find that the misbalance depends on the aspect angles $\alpha_{6}$ of the opposite sub-walls:

$$
H_{d w}=M_{s}<\Sigma \alpha_{t i}>/ \pi
$$

where $\langle>$ denotes average over the 'viewpoints'. It may now be observed that (i) in the 'flat' limit, the intra-layer $\alpha_{\text {to }}$ is comparable to all others, so that interlayer fields prevail in multilayers, and all $\alpha_{\mathrm{ti}}$ approach f. $\alpha_{\mathrm{pi}}$ (the aspect angles of $p=t+s$ ), which accounts for the average (UE) scaling of $\mathrm{H}_{\mathrm{dw}}$; (ii) on diminishing the width, D, the inter-layer angles (and fields) are maximum when the horizontal and vertical distances are equal; thus the interlayer contributions have a maximum in the square-domain range; (iii) in the sharp limit, the intralayer angles (fields) dominate $\left(\mathrm{H}_{\mathrm{dw}}\right.$ finally saturates at the single-layer value $M_{s}$ ). The 'sharp' limit has no practical relevance in recording; in

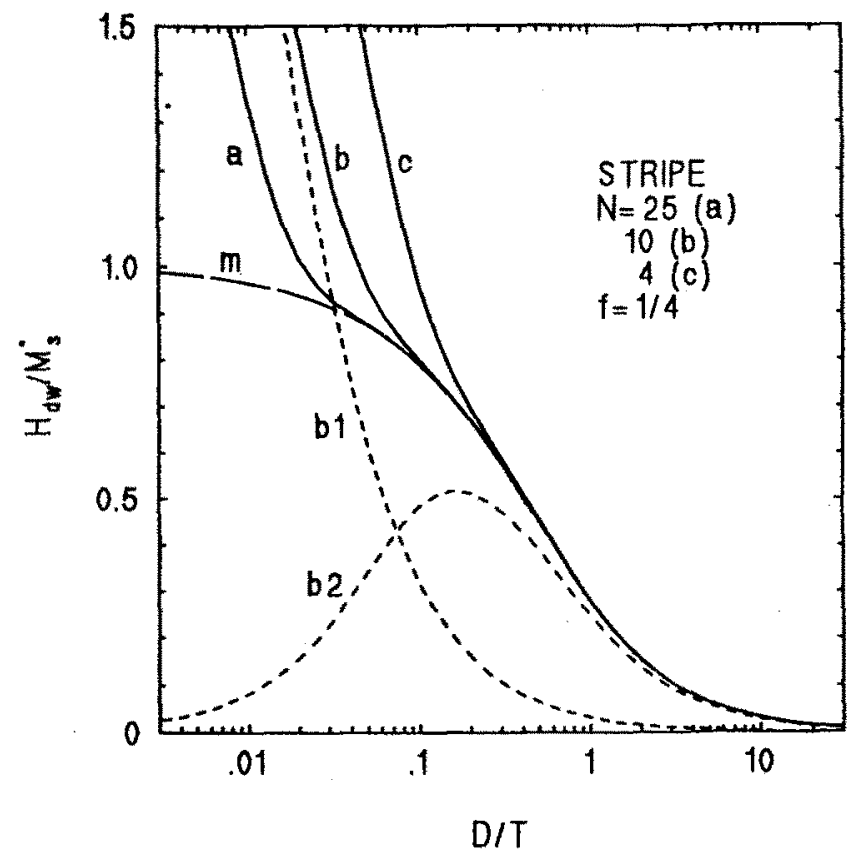

Fig. 3 Mean field $\mathrm{H}_{d w}$ at wall of a stripe domain of diame ter $\mathrm{D}$ in ML of thickness $T$ (solid). $M_{s}{ }^{*}$ is the mean magnetization. (b1) and (b2) are the intra- and inter-layer constituents of the (b) line, $(m)$ is $\mathbf{H}_{d w}$ in uniform equivalent film. the square range, some corrections to the UE results are only needed in computation of precise $\mathrm{ML}$ model values [3].

Surface tension in the domain boundary tends to shrink cylindrical (or any curved) boundaries; it is characterized by the wall energy density, $\sigma$ or, relatively to the magnetostatic energy density, by the characteristic 'material length' of the magnetic layers, $l$ $=\sigma /\left(\mu_{0} \mathbf{M}_{\mathrm{s}}{ }^{2}\right)$. It is practical to use averaged parameters $[3,4], I^{*}=\sigma^{*} /\left(\mu_{0} \mathbf{M}^{* 2}\right)=l / f$ with $\sigma^{*}=f \sigma$. The total effective field acting on cylindrical walls in ML is then given by equation

$$
\mathrm{H}_{\mathrm{eff}} / \mathrm{M}_{\mathrm{s}}^{*}=\mathrm{H}_{\mathrm{dw}} / \mathrm{M}_{\mathrm{s}}^{*}-l^{*} / \mathrm{D}
$$

In hard films, $\mathrm{H}_{\text {eff }}$ is not necessarily zero but should be kept well below the value of coercivity.

\section{EQUILIBRIUM STRIPE ARRAYS}

Equilibrium configurations are of less direct interest to recording, but the domain sizes in equilibrium may serve as an important guide for two purposes - they should not be exceeded too much in artificial structures, and they allow to estimate, from equilibrium domain observations, the wall energy density.

The energy and field balancing in the regular (periodic) stripe model differs in principle from the case of isolated domains; as both the surface tension in walls and the magnetostatic mean $\mathbf{H}_{d w}$ at wall positions vanish (in zero applied field), minimum total energy is sought with respect to the number of domains (in the

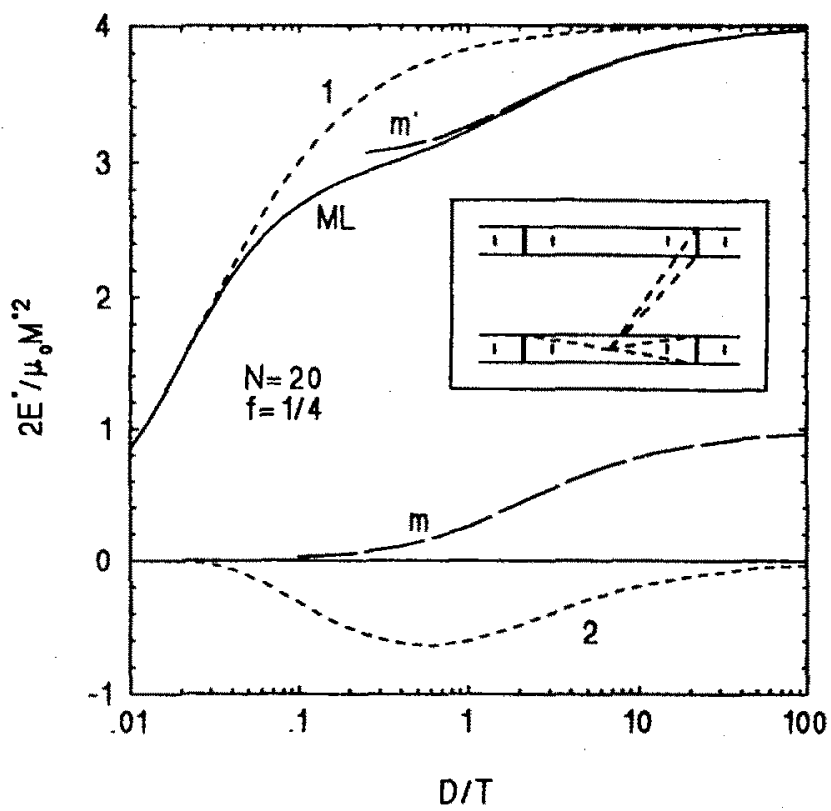

Fig. 4 Mean energy density $\mathrm{E}$ in periodic stripe model in a $\mathrm{ML}$ (solid) and uniform equivalent $(\mathrm{m})$. (1) and (2) are the intraand inter-layer constituents of the ML line. 


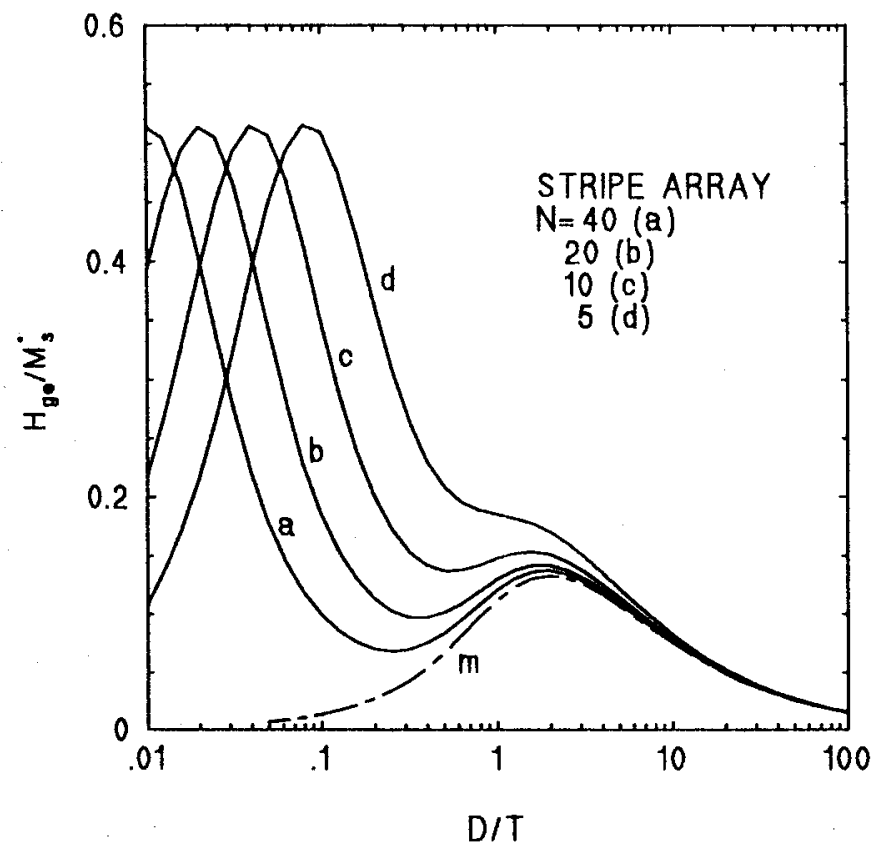

Fig. 5 Left-hand side of eq.(4) for ML of filling $f=1 / 4$ and various bilayer numbers $N$; $(m)$ is for uniform equivalent film.

theory of domain 'phase' transitions [7] such energy derivatives are treated as chemical potentials).

The equation of 'global' equilibrium may be written in a form formally analogous to ' $H_{\text {eff }}=0$ ' in eq.(3),

$$
\partial\left(\mathrm{E}_{\mathrm{d}}^{*} / \mu_{\mathrm{o}} \mathrm{M}_{\mathrm{s}}^{* 2}\right) / \partial(\ln \mathrm{D})=l^{*} / \mathrm{D}
$$

where $\mathrm{E}_{\mathrm{d}}^{*}$ is the magnetostatic energy density averaged over the total ML volume, usually computed in terms of Fourier expansions $[2,3]$.

The results of such calculations for ML are in Fig.4: as in the preceding plots, $\mathrm{E}_{d}{ }^{*}$ is compared with the UE result (bold-dash line $\mathrm{m}$ ) and decomposed into intralayer and interlayer contributions (light-dashed 1 and 2). The main features of the $E_{d}{ }^{*}$ plot are, (i) the absolute value saturates at $\mu_{0} M_{s}^{* 2} / 2 f$ in the 'flat domain' limit, but (ii) the rate of change with $D$ copies the UE behavior, as evidenced by perfect nesting with the $m$ line (show by its shift to $m$ ' in the figure), and (iii) approach to 'decoupled-layer' behavior (line 1) in the (rather academic) case of narrow domains. This can be understood intuitively on the basis of 'angular' argu ments similar to those for isolated stripes, schematized in the inset of Fig.4: the value of $E_{d}^{*}$ is mainly determined by the amplitude of $\mathbf{H}_{d}$ in the domain centres, since it vanishes at wall positions; in the 'flat' case, the large negative value of $H_{d}$ is dominated by the wide angle including the sub-domain top but its rate of change with $D$ is equivalently derived from the sharp angles including the walls of all subdomains in the stack.

Fig.5 shows plots of the 1.h.s. of eq.(4), called $\mathrm{H}_{\mathrm{ge}} / \mathrm{M}_{\mathrm{s}}^{*}$ in formal analogy to eq.(3).

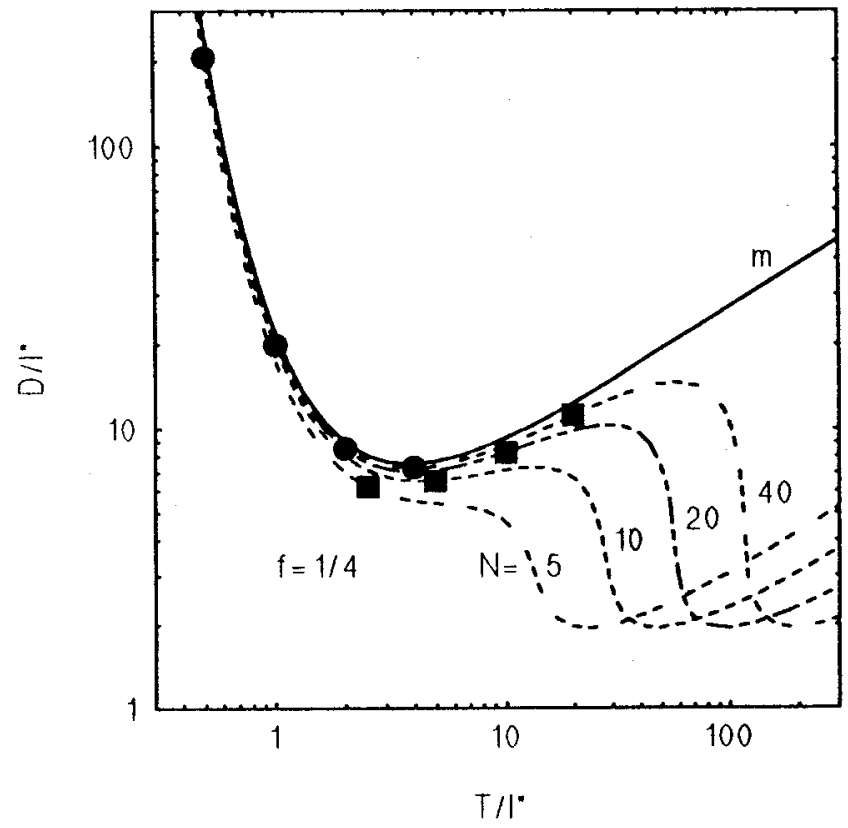

Fig.6 Equilibrium stripe width D vs. ML thickness T: dashed lines are 'inflation plots' at fixed $f$ and $N$; open points are 'stacking plots' for the 4 values of $N$, at fixed $f$ and $t=0.1$ ( and $0.5(\omega)$.

It is now practical to scale $\mathrm{D}$ as well as $\mathrm{T}$ by $l$ [8] and obtain plots of the solutions of eq.(4), as in Fig.6. Here, the solid line is the UE solution [8]. The open points are the predictions for two sequences of $\mathrm{N}$ values, at fixed $f$ and fixed (postulated) $t / l$ parameters, which corresponds to the usual ML production. The dashed lines corespond to 'inflation' (ideal) experiment, increasing $T$ and $t, s$ at the same rate, at fixed $f, N$, as in all the preceding plots; these lines are 'universal' , i.e., independent on the characteristic length, $l$. They also show that the severe departures from the UE behavior predicted [4] fortunately only occur in ranges irrelevant for practical recording. Experimental equilibrium domain sizes in CoPd ML $[4,6]$ are all on the 'flat' side (D> T), close to the UE approximation.

\section{WALL ENERGY DENSITY}

The material lengths $l$ and $l$ are usually assumed to be proportional to the Landau-Lifhitz value of wall energy density, $\sigma_{\mathrm{LL}}=4 \sqrt{ }\left(\mathrm{AK}_{\mathrm{u}}\right)$ with $A$ and $K_{u}$ for the exchange stiffness and (perpendicular) uniaxial anisotropy constants. This is, however, only good for very thick films with wall magnetization parallel to wall plane (Bloch orientation) and flux-closing regions with wall magnetization mostly perpendicular to wall plane (Néel orientation) limited to relatively very thin surface layers [9].

Ploessl et al. [5] recently drew attention to the fact that the absence of exchange coupling between sublayers may support fully Néel-wall-like orientations of wall moments with significant flux closure inside the 


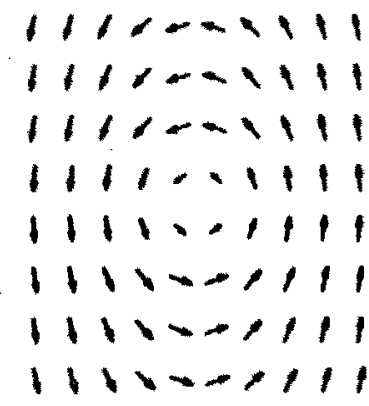

(a)

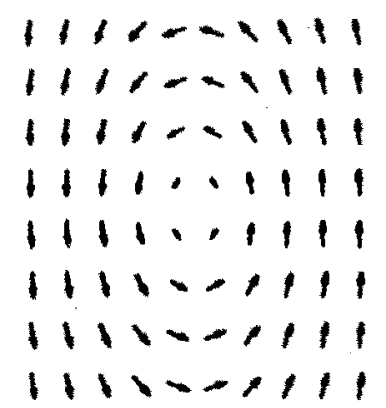

(c)

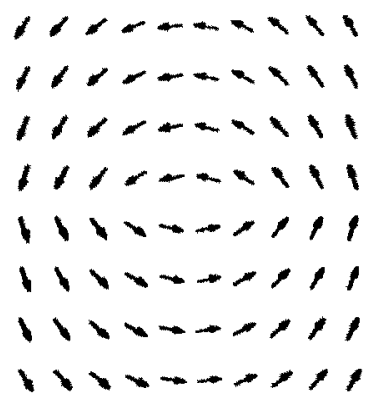

(b)

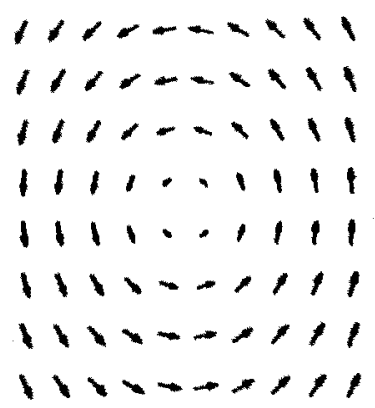

(d)
Fig.7 Self-consistent wall configurations for $Q=2(a, c)$ and $Q=1.2(b, d)$ with interlayer exchange zero $(a, b)$ and $5 \%(c, d)$. Rows represent magnetic sublayers (vertical period $=2 \mathrm{~nm}$ ).

walls (as in Fig.7). In that case, we may also expect significant decrease of the wall energy due to saving on magnetostatic energy. A crude estimate based on local approximation for the dipolar energy density yields for the mean wall energy density

$$
\sigma_{d}=4 V\left(A K_{e f f}\right)=\sigma_{L L} V(1-1 / Q)
$$

with "effective anisotropy" $\mathrm{K}_{\mathrm{eff}}=\mathrm{K}_{\mathrm{u}}-\mu_{\mathrm{o}} \mathrm{M}^{2} / 2$ and 'quality' factor $\mathrm{Q}=2 \mathrm{~K}_{\mathrm{u}} / \mu_{\mathrm{o}} \mathrm{M}_{\mathrm{s}}^{2}$.

It is also interesting to note that approximate account for flux-closing tendency by the $\mu$ method $[10]$, used by Kooy and Enz [11] for stripe domains in uniform films to extend the earlier results [8] to low-Q materials, predicts slightly increasing $D$ (with decreasing $Q$ ) in thick films (for $D<T$ ) [11], but decreasing $D$ in thin films (D> T), as shown in Fig. 8. Qualitatively, in thick films flux-closure lowers the surface [12] strayfield energy instead of need for more domains, but in thin films stray-field energy is lowered in walls only, giving more advantage with more domains. On the 'flat domain' side of Fig.8 (D $>2 T)$, the finite-Q (dashed) lines may be shifted to the $Q=\infty$ (solid) line using renormalized $l$, which correponds to wall energy reduction analogous to the ML results presented below, but overestimated because competition with the exchange energy is neglected in the $\mu^{*}$ method.

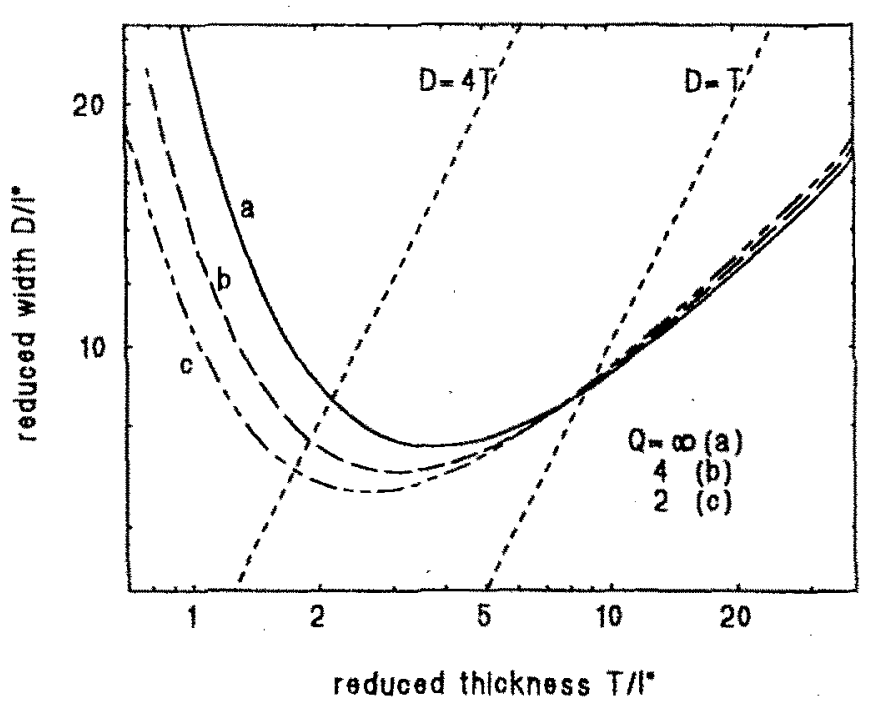

Fig, 8 Equilibrium stripe width D vs. film thickness $T$ in the Kooy-Enz model [12] for uniform films (where flux closure mostly affects film surface and whole walls, in the limits of large and small $T$, respectively).

Micromagnetic discrete modelling of relaxed wall configurations in ML with magnetostatics included was performed following LaBonte [13] (as also described by Ploessl et al.[5] for ML). Straight walls in ML are simulated by rows of 100 uniformly magnetized squarerods in each magnetic sublayer (at $t=0.5 \mathrm{~nm}$, the cell dimension equal to $t$ was only $1 / 10$ of the exchange length). Their magneto-static interaction is included self-consistently in the relaxation process; the constant field of the remainder of the stripe-domain structure is also added (unrelaxed). Only 'flat' domains (D>2T) were so far considered. Exchange interaction between magnetic sublayers was zero or $5 \%$ of 'lateral' exchange.

Typical relaxed configurations (near wall centers) are shown in Fig. 7, for $N=8$ and $f=1 / 4$ (the vertical period $t+s$ is $2 \mathrm{~nm}$, the horizontal distance of 'spins' shown is $1.5 \mathrm{~nm}$ ) Only projections of magnetization on the plane perpendicular to wall axis (length) are shown: note that magnetization is confined to this plane (Néel orientation) only for low $Q$ and zero inter-layer exchange [5], but in other cases the 'swirl' at the wall center has a Bloch-wall-like component.

Wall energy density $\sigma$ was also measured on the model, with the magnetostatic contribution defined as the difference between the dipolar energies of the relaxed configuration and of that assumed in the stripe domain model ('up' and 'down' magnetization strictly perpendicular to the film plane). Typical results are in Fig. 9, for $f=1 / 4$ and $N=8(0)$ and $16(n)$; the increase of $\sigma$ when inter-layer exchange (of $5 \%$ ) is added is also shown $(t)$. Closeness of these micromagnetic results 


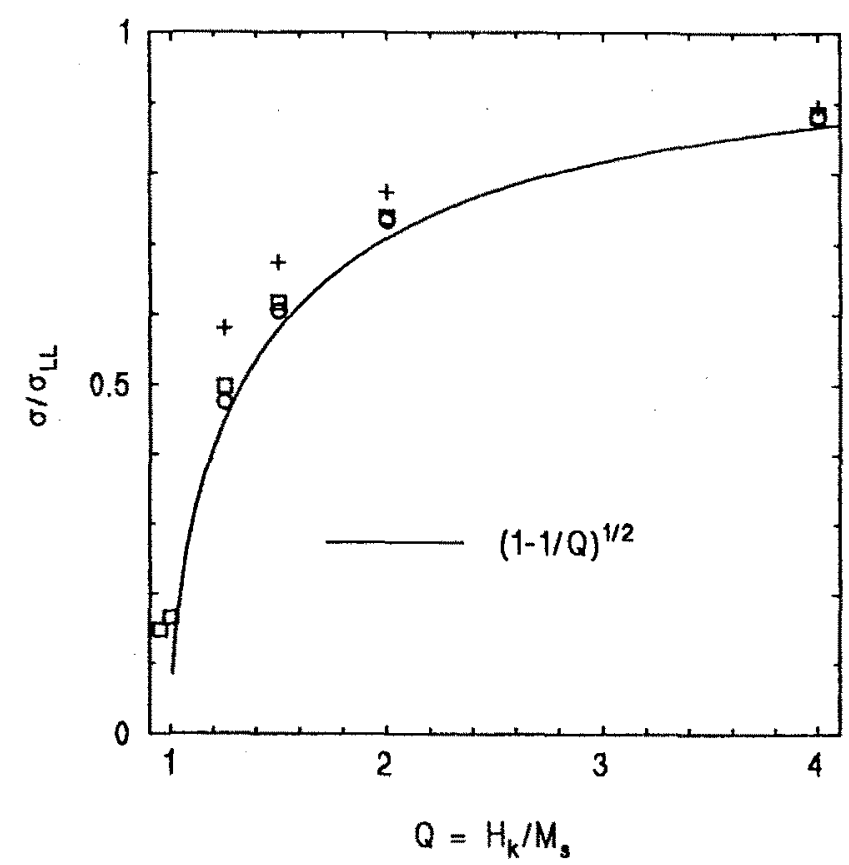

Fig.9 Domain wall energy density $\sigma$ reduced by flux closure in ML's with varying 'quality' factor $Q$ and zero $(0, D)$ or $5 \%$

$(+)$ inter-layer exchange ( $\sigma_{\mathrm{LL}}$ is the Landau-Lifshitz value).

to the estimate (5), based on local approximation for the magnetostatic energy (solid line), shows that interlayer magnetostatic interactions determine the global flux-closing spin configuration [5] but do not significantly affect the total energy.

At low $Q$ values (below about 0.8 ), the computed $\sigma$ is negative, which is a real effect indicating instability of perpendicular magnetization in the domains [14] (some of the earlier wall simulations [5], where wall energy was not measured, were in fact performed in this instable range).

The 'single-wall' approximation in the above model invalidates the results in the 'square' domain range (for $D \approx T$ ) where the 'wall energy density' depends on $D$, the classical balance eq. (4) with constant $l^{*}$ cannot be used and the total energy of the periodic micromagnetic model should also be minimized with respect to $D[15]$. However, the above results for 'flat' domains indicate that significant wall energy reduction must be expected in presently available $\mathrm{ML}$, with $\mathrm{Q}$ factors mostly between 1 and 2 .

\section{EXPERIMENTS IN CoPd}

Small effective fields acting on walls of flat domains, combined with high coercivity, are favorable to stability of artificial patterns, but approach to 'global equilibrium' assumed in the array balance (4) requires special care (ac demagnetization) in experiments.

Domains in nearly demagnetized states were recently observed $[4,6]$ on a series of polycrystlline (sput tered) ML prepared in MESA Institute (University of

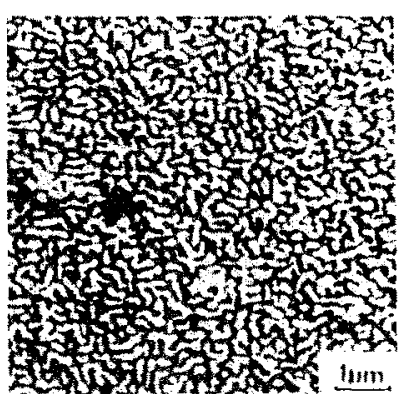

No. 1

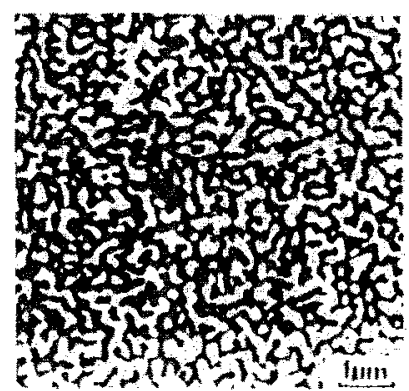

No. 3

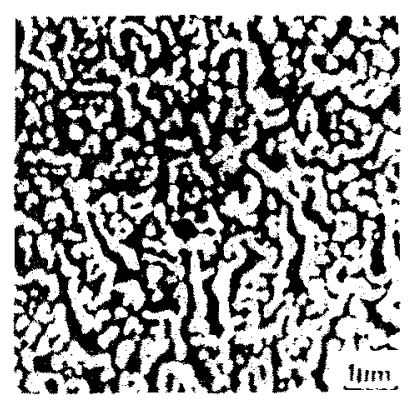

No. 2

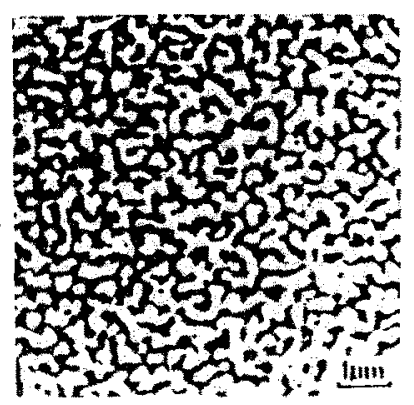

No. 4
Fig.10 Demagnetized domain structures in CoPd ML imaged by CSEM (cf. Tab.1 for ML parameters).

Twente) [16]. Typical domain patterns obtained by SEM from dried Bitter colloid decoration (CSEM [17]) are in Fig. 10; analogous pictures obtained by MFM were also reported [6]. The average domain widths are given in Tab.1 along with other film parameters [16].

Table 1. Experimental results on CoPd multilayers $[16,4,6]$

\begin{tabular}{lccccccccc}
\hline $\begin{array}{l}\text { sample } \\
\text { no. }\end{array}$ & $\mathrm{t}$ & $\mathrm{N}$ & $\mathrm{f}$ & $\begin{array}{r}\mathrm{T} \\
\mathrm{nm}\end{array}$ & $\begin{array}{r}\mathrm{D} \\
\mathrm{nm}\end{array}$ & $\begin{array}{c}\boldsymbol{l} \\
\mathrm{nm}\end{array}$ & $\begin{array}{c}\sigma \\
\mathrm{mJ} / \mathrm{m}^{2}\end{array}$ & $\mathrm{Q}$ & $\begin{array}{r}\mathrm{H}_{\mathrm{c}} \\
\mathrm{kA} / \mathrm{m}\end{array}$ \\
\hline 1 & 0.85 & 25 & 0.37 & 57.4 & 90 & 12.1 & 20 & 1.1 & 36 \\
2 & 0.43 & 25 & 0.13 & 83.9 & 145 & 21.3 & 9 & 2.0 & 111 \\
3 & 0.45 & 25 & 0.24 & 46.5 & 135 & 18.0 & 22 & 1.4 & 96 \\
4 & 0.23 & 25 & 0.13 & 41.0 & 190 & 21.4 & 22 & 1.4 & 152 \\
5 & 0.40 & 7 & 0.27 & 10.5 & 230 & 11.0 & 8 & & 55 \\
\hline
\end{tabular}

Fig.11 shows comparison of these results with the stripe-model predictions: the experimental input is actually the $\mathrm{D} / \mathrm{T}$ ratio (straight lines), the dashed lines are computed from the model ('inflation' plots of Fig. 6 with the experimental structure parameters; the solid line is the 'universal' UE plot). Evaluation of $l$ from this kind of fitting yields values of the wall energy density $\sigma$ mostly around $20 \mathrm{~mJ} / \mathrm{m}^{2}[4,6]$, close to the classical $\sigma_{L L}$ with $K_{u}$ measured [16] and the exchange constant $A$ near the bulk $C o$ value. These energy estimates are substantially (more than twice) higher than analogous estimates obtained for $\sigma$ in CoPt ML $[3,18]$. In particular, the energy reduction expected in the low-Q sample (no.1) could not be confirmed; on the 


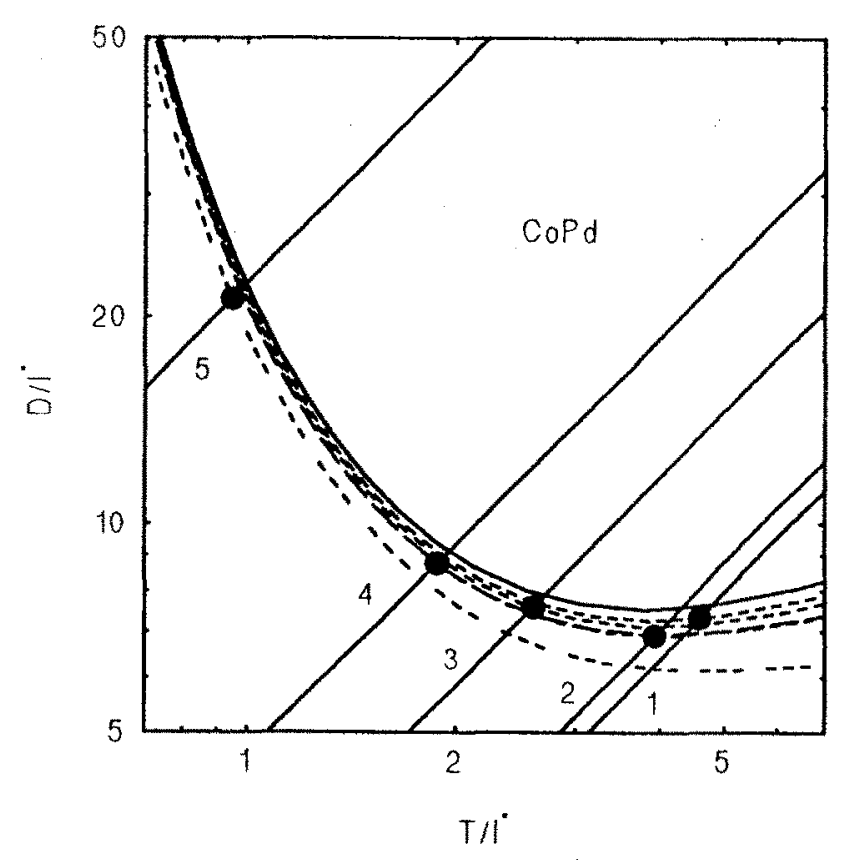

Fig.11 Comparison of theoretical (dashed as in Fig. 6) and experimental (points) domain wall width in CoPd ML.

other hand, the low value of $\sigma$ obtained for sample no. 2 may be related to its large Pd thickness $(29 \mathrm{~nm}$, twice more than in other samples). Thus, along with other deficiencies of the model, we feel particularly concerned about the actual magnitudes and distribution of exchange interactions in the CoPd multilayers, particularly across the Pd spacers; some (so far weak) indication of their positive role in enhancing the wall energy might be found from this kind of experiments.

\section{ACKNOWLEDGEMENT}

Fruitful discussions and permission to quote unpublished preliminary results by J. Šimšová, P.deHaan and J.C. Lodder are most gratefully acknowledged.

\section{References}

[1] A. Suna, Journ.Appl.Phys.59, 313 (1986)

[2] H.J.G. Draaisma and W.J.M. deJonge, Journ.Appl.Phys. 62, 3318 (1987)

[3] S. Honda, Y. Ikegawa and T. Kusuda, Journ. Mag. Mag. Mater. 111, 273 (1992)

[4] V. Kamberský, P. deHaan, J.C.Lodder, J. Šimsová and R. Gemperle, IEEE Trans. Mag. 29, 784 (1993)

[5] R. Ploessl, J.N. Chapman, M.R. Scheinfein, J.L. Blue, M. Mansuripur and H. Hoffmann, Journ.Appl.Phys. 74, 7431 (1993)

[6] J. Šimsová, R. Gemperle, V. Kamberský, S. Porthun, P. deHaan and J.C. Lodder, IEEE Trans. Mag. 30, 784 (1994)

[7] E.A. Zavadskii and V.A. Zablotski, Phys.Stat. Sol. (a) 112, 145 (1989)

[8] Z. Málek and V. Kamberský, Czech.Journ. Phys. 8, 416 (1958)

[9] A. Hubert, Journ.Appl.Phys. 46, 2276 (1975)

[10] H.J. Williams, R.M. Bozorth and W. Shockley, Phys. Rev. 75, 155 (1949)

[11] C. Kooy and U. Enz, Philips Res.Repts. 15, 7 (1960)

[12] C. Kittel, Phys. Rev. 70, 965 (1946)

[13] A.E. LaBonte, Journ. Appl. Phys. 40, 2450 (1969)

[14] M. Labrune and J. Miltat, IEEE Trans.Mag.26, 1521 (1990); Journ.Mag.Mag.Mater. 104, 241 (1992)

[15] J. Kaczér, M. Zelený and P. Šuda, Czech. Journ. Phys. B 13, 579 (1963)

[16] P. deHaan, Q. Meng, T. Katayama and

J.C. Lodder, Journ.Mag.Mag.Mater. 113, 29 (1992)

[17] J. Šimšová, R. Gemperle and J.C. Loder, Journ.Mag.Mag.Mater. 95, 85 (1991)

[18] D.M. Donnett, J.N.Chapman, H.W. van Kesteren and W.B. Zeper, Journ.Mag.Mag.Mater. 115, 342 (1992) 